\title{
Sensitivity Visualization of Circulation under Congestion and Blockage
}

\author{
Gabriel Wurzer, Matthias Ausserer, Harry Hinneberg, Christa Illera and \\ Andrea Rosic
}

Vienna University of Technology

Vienna, Austria

Corresponding author: wurzer@iemar.tuwien.ac.at

\begin{abstract}
In the context of circulation design for large buildings (e.g. hospitals, airports), the question of sensitivity of the path network against congestions and blockages naturally arises. To date, the answer to this question would require planners to use a simulation package, which is, however, almost never done in the early stages of building design. We therefore propose a novel visual planning tool that enables architects to estimate the impact of disturbances on the building circulation without having to use a simulation package. Our approach is integrated into a common CAD system and visualizes changes in the path-time relationship of adjacent functional areas under the effects of impeded accessibility.
\end{abstract}

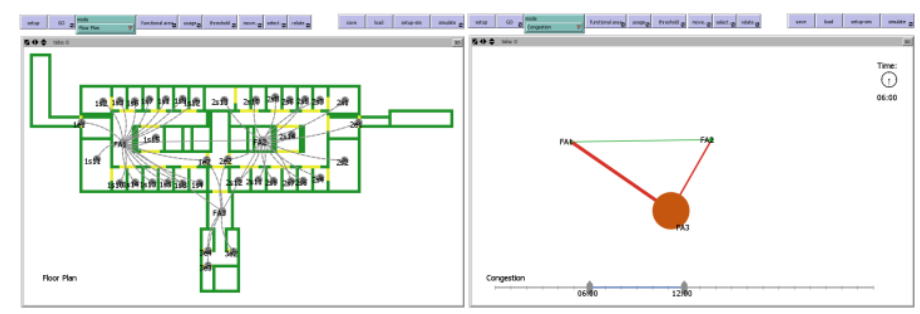

Fig. 1. Based on an attributed floor plan (left), we perform a visualization of sensitivity against congestion and blockages (right). Nodes are used for functional areas and edges for circulation. The colour of each edge gives the sensitivity.

\section{Introduction}

Our planning tool is a graph which visualizes functional areas as nodes and connections between functional areas as edges (refer to Fig. 1, right). A functional area is a collection of pedestrian sources and thresholds (either leading to the next functional area or being exits). Each pedestrian source models the usage of a space in a time span t0 to $\mathrm{t} 1$. Usage data is of high interest in complex buildings which 
are designed around pre-determined processes (e.g. hospitals, airports). Our goal is to try to visualize flows along the circulation (both in and between functional areas), the target audience being architects who want to get "insight, not data" (as once proposed by Hamming).

Our program is integrated with a CAD system. As starting point, we take a basic CAD drawing that depicts walls and thresholds as input. This drawing is imported as raster image with a resolution of $0.5 \mathrm{~m}$, and must then be attributed with functional areas, pedestrian sources and threshold points in a pre-step (Fig. 1, left). A cell-space simulation then computes flows from the sources to the exits in the set time span, giving us:

- the number of people crossing each circulation between two functional areas

- the duration of travel along this circulation

This information forms the basis for our visualization, which will now be described.

\section{Visualization of sensitivity against congestion}

The thickness of each edge (see Fig. 1, right) depicts the throughput of a circulation, i.e. the number of people crossing it in the set time-span. The same goes for the size of each functional area node, which tells the amount of people that have entered it. We have opted use the absolute value of the throughput, scaled by a user-defined constant, which gives us good results.

The duration of travel along the circulation is depicted as edge color. However, we do not use this value directly, but first look at the whole time-span that is set. It is the difference between the maximum travel times that is taken gives us a measure of congestion in both functional areas (nodes) and the circulation in between them (edges), which we map to a red-green color gradient. In this sense, attaining "thick green lines" (high throughput, little sensitivity against congestion) is the preferable goal. The mapping of differences in travel times onto colors is obtained by taking ranges and mapping these to discrete colors (classification of absolute values).

\section{Visualization of sensitivity against blockages}

In a pre-step, the simulation runs through the set time span and records, for each functional area node and circulation edge, the maximum travel time. Then, the algorithm subsequently removes an edge from the circulation graph and performs the simulation again. The difference between the recorded and the current maximum duration time gives a measure of the sensitivity against blockages in the chosen time span, which is again mapped to a red-green color scale in the same fa- 
shion. The throughput is visualized in the same way as before, as thickness of circulation edges and functional area nodes.

\section{Underlying simulation}

Our simulation uses a raster image that was exported from the CAD drawing as input into a cell-space simulation algorithm, which performs the actual work of generating the data that is to be visualized. Furthermore, we require that, for each pedestrian source, there is usage data (in the form of a spreadsheet table). This usage data tells the simulation engine how many agents to generate at a given time in which pedestrian source area (see Fig. 2). The agents are then simulated using the model of Blue and Adler [1] which is extended with a higher-level exit route choice function, in order to support choosing exits on the bases of functional areas. Furthermore, the extension is also responsible to disallow access to functional areas that are currently marked as "blocked".

Each performed simulation run measures when and how many agents cross area borders. These resulting values are recorded for later use in the visualization. When crossing thresholds, agents must choose their next target using our extension algorithm that considers adjacent functional areas. If there is such an adjacent functional area, the agent resets his internal clock and crosses the functional area to find an exit. In due course, the number of agents crossing the circulation is incremented by one. Upon reaching the exit, the agent records the total time of travel for the circulation he has just crossed, and the choosing of the next functional area continue. If there is no further functional area to go to, the agent is taken out of the simulation (the exit can then be considered as safe area).

\section{Previous Work}

Our work employs usage data of functional areas to aid the planning process. Previous work in this context recorded and simulated building user's activities [2] in order to assess the building design's performance. Extending CAD systems with user activities has also previously been researched in [3][4][5]. Furthermore, our work focuses on providing meaningful visualizations of simulation data, which have been considered in [6][7]. From the view of pedestrian dynamics community, architectural considerations have been previously brought forward by [8][9]. 


\section{Conclusions}

We have brought forward the idea of a novel diagram that lets an architect assess the effects of congestions and blockages on the planned circulation. Our concept is integrated into a regular CAD system and can be used during the early stages of building design. We are confident that, using our approach, architects can further improve the circulative system (i.e. add redundant paths, or increase capacity) in order to design safer.

We are aware of several points that our approach does not address and that require a justification. First of all, our underlying simulation is rather simple (for example, we have not considered taking any response times into account). In the context of this paper, simulation is merely a basis for gathering data, which is to be fed into a higher-level visualization. It is clear that more elaborate forms of simulation algorithms that have been in existence for a long time could be added. As our prototype will be published under an open-source license, we encourage interested researchers to do so.

\section{References}

1. Dijkstra J., Timmermans, H. and Vries, B.: Virtual Reality Based Simulation of User Behavior within the Built Environment to Support the Early Stages of Building Design, CIB Conference (2001)

2. Ekholm, A. and Fridqvist, S.: Concepts of Space in Computer Based Product Modelling and Design, eCAADe Conference (1997)

3. Wurzer, G.: Systems - Constraining Functions through Processes (and Vice Versa), eCAADe Conference (2009)

4. Wurzer, G., Loffreda, G., Fioravanti, A. and Trento, A.: Function versus Action, submitted to eCAADe Conference (2010)

5. Wurzer, G.: Visualizing the Human Form for Simulation and Planning, Pedestrian and Evacuation Dynamics Conference (2008)

6. VDI 3633 Part 11: Guideline on Simulation and visualization, Verein Deutscher Ingenieure (2005)

7. Illera, C., Fink, M., Hinneberg, H., Kath, K., Waldau, N., Rosic, A. and Wurzer, G.: NO_PANIC - Escape and Panic in Buildings, Pedestrian and Evacuation Dynamics Conference (2008)

8. Wurzer, G., Hinneberg, H., Illera, C., Swoboda, S. and Jonas, A.: Architectural Patterns Enabling Reconfigurable Exit Routes for Complex Buildings, Traffic and Granular Flow (2009) 